\title{
A Comparative Study Analyzing Differences in Factors Related to Childbirth Satisfaction between Primiparous and Multiparous Mothers
} Maria Belen Conesa Ferrer ${ }^{1 *}$ and Marcos Camacho Avila ${ }^{2}$

${ }^{1}$ Department of Nursing, Universidad de Murcia, Spain

${ }^{2}$ University Hospital of Torrevieja, University of Almeria, Spain

\section{Retraction Note:}

The article entitled "A Comparative Study Analyzing Differences in Factors Related to Childbirth Satisfaction between Primiparous and Multiparous Mothers" has been accepted for publication in the Journal of Nursing \& Care considering the statements provided in the article as personal opinion of the author which was found not having any conflict or biasness towards anything. As the article was a perspective one, information provided by the author was considered as an opinion to be expressed through publication.

Publisher took decision to make the article online solely based on the reviewers suggestion which considered the article not but a personal opinion of the author. However, it is found that the author have some personal concerns and issues, therefore, being retracted from the journal. 